\title{
EVERY ATTRACTOR OF A FLOW ON A MANIFOLD HAS THE SHAPE OF A FINITE POLYHEDRON
}

\author{
BERND GÜNTHER AND JACK SEGAL
}

(Communicated by James E. West)

\begin{abstract}
It is shown that the class of compacta which can occur as attractors of continuous flows on topological manifolds coincides with the class of finite dimensional compacta having the shape of a finite polyhedron.
\end{abstract}

\section{THE SHAPE OF ATTRACTORS}

Let $X$ be a locally compact, separable Hausdorff space. By a flow we mean a continuous group action by the reals on $X, X \times \mathbb{R} \rightarrow X$. The image of $(x, t) \in$ $X \times \mathbb{R}$ under the operation is denoted $x^{t}$; by definition we have $x^{s+t}=\left(x^{s}\right)^{t}$ and $x^{0}=x$. For $A \subseteq X$ and $M \subseteq \mathbb{R}$ we write $A^{M}:=\left\{x^{t} \mid x \in A, t \in M\right\}$. A subset $A$ of $X$ is said to be positively invariant if $A^{[0, \infty[} \subseteq A$, it is invariant if $A^{t}=A$ for every $t \in \mathbb{R}$. We emphasize that the dynamical systems under consideration are continuous, i.e., indexed by $\mathbb{R} ;$ if $\mathbb{R}$ is replaced by the integers $\mathbb{Z}$ in the definition above we speak of a cascade. We will see that cascades behave entirely different in the situations we have in mind.

An attractor in a flow is a nonempty, invariant, compact subset $A \subseteq X$, which admits a neighborhood $U$ in $X$, such that for each neighborhood $V$ of $A$ in $X$ there is a real number $t \geq 0$ with $U^{[t, \infty[} \subseteq V$. This definition is equivalent to the ones given in $[1$, p. $199 ; 11$, p. 8]. A neighborhood $U$ with the property above will be called a domain of attraction for $A$.

For cascades as well as for flows it is hitherto unknown which type of homeomorphism an attractor can have. The present paper solves this problem for flows. Our main result, Corollary 4, may be viewed as an extension of the classical Poincaré-Bendixson Theorem [7, p. 248].

Theorem 1. (a) If $A$ is an attractor of a flow on $X$, then $A$ has a compact neighborhood $U$ such that $U \backslash A$ is homeomorphic to $\partial U \times[0, \infty[$ with $\partial U$ mapped onto $\partial U \times\{0\}$.

Received by the editors January 27, 1992.

1991 Mathematics Subject Classification. Primary 54C56, 54H20, 58F12.

Key words and phrases. Dynamical systems, attractors, shape, complement theorem.

This paper was started while the first author visited the University of Washington, supported by a DFG fellowship. He also acknowledges helpful conversations with Peter Mrozik. The referee's comments provided further improvement. 
(b) We suppose $A$ is a compact subset of $X$, for which there exist a compact neighborhood $U$ and a homeomorphism $h: U \backslash A \approx \partial U \times[0, \infty[$ with $h(\partial U)=\partial U \times\{0\}$, such that $h^{-1}: \partial U \times[0, \infty[\rightarrow U$ satisfies the local Lipschitz condition $(\mathrm{L})$. Then $A$ is an attractor of a suitable flow on $X$.

We consider a suitable metric $d$ on the compact metrizable space $U$. Then for each point $\left(y_{0}, t_{0}\right) \in$ $\partial U \times\left[0, \infty\left[\right.\right.$ there exist a neighborhood $V_{0}$ of $y_{0}$ in $\partial U$, a neighborhood $W_{0}$ of $t_{0}$ in $[0, \infty[$, and a number $K\left(y_{0}, t_{0}\right)>0$ such that the inequality $d\left(h^{-1}(y, s), h^{-1}(y, t)\right) \leq K\left(y_{0}, t_{0}\right) \cdot|s-t|$ holds whenever $y \in V_{0}$ and $s, t \in W_{0}$.

Proof. (a) Let $V$ be a compact domain of attraction for $A$. We may assume that $V$ is positively invariant, because we can replace it by $\left\{x \in V \mid x^{[0, \infty[} \subseteq V\right\}$. Then $A$ is an isolated invariant set with $V$ as the isolating neighborhood [10, p. 380]. By [10, Theorem 5.2] there is a Lyapunov function $\Phi: V \rightarrow \mathbb{R}$, i.e., a continuous map with $\Phi \equiv 0$ on $A$ and $\Phi\left(x^{t}\right)<\Phi(x)$ for all $x \in$ $V \backslash A$ and $t>0$. Since $x^{t}$ approaches $A$ for $t \rightarrow+\infty, \Phi\left(x^{t}\right)$ must decrease towards 0 , so that $\Phi \geq 0$ on $V$ with $A=\Phi^{-1}(0)$. We choose a number $0<\alpha<\min \{\Phi(x) \mid x \in \partial V\}$, and for each $x \in V \backslash A$ we set $f(x)$ equal to the unique number with $\Phi\left(x^{-f(x)}\right)=\alpha$. Such a number always exists, because the set $x^{\mathrm{J}-\infty, 0]}$ cannot be entirely contained in the domain of attraction $V$. To show that $f: V \backslash A \rightarrow \mathbb{R}$ is continuous we consider $\varepsilon>0$ and get $\Phi\left(x^{\varepsilon-f(x)}\right)<\alpha<\Phi\left(x^{-\varepsilon-f(x)}\right)$. There is a neighorhood $W$ of $x$ in $V \backslash A$ with $\Phi\left(y^{\varepsilon-f(x)}\right)<\alpha<\Phi\left(y^{-\varepsilon-f(x)}\right)$ for all $y \in W$, and this implies $-\varepsilon-f(x)<$ $-f(y)<\varepsilon-f(x)$ for $y \in W$.

We set

$$
U:=\{x \in V \mid \Phi(x) \leq \alpha\} .
$$

Then $U$ is a compact, positively invariant neighborhood of $A$ with $\partial U=$ $\Phi^{-1}(\alpha)$; furthermore, we have $f \geq 0$ on $U \backslash A$. Hence we can define $h$ : $U \backslash A \rightarrow \partial U \times\left[0, \infty\left[\right.\right.$ by $h(x):=\left(x^{-f(x)}, f(x)\right) . h$ is a homeomorphism with $h^{-1}(y, t)=y^{t}$.

To prove the converse we need the following lemmata:

Lemma 1. There is a flow $\mu:[0, \infty[\times \mathbb{R} \rightarrow[0, \infty[$ on $[0, \infty[$ satisfying the following properties:

(a) 0 is a restpoint.

(b) $\lim _{t \rightarrow+\infty} \mu(x, t)=\infty$ uniformly on each interval of the form $[\eta, x[$ with $\eta>0$.

(c) $\forall \varepsilon>0, \eta>0 \exists \beta>0 \forall x>\beta, t \in \mathbb{R}:|t|<\eta \Rightarrow|\mu(x, t)-x|<\varepsilon$.

Lemma 2. The homeomorphism $h$ in Theorem 1 (b) can be altered such that the Lipschitz condition (L) is satisfied globally, i.e., with $V_{0}=\partial U, W_{0}=[0, x[$ and $K\left(y_{0}, t_{0}\right)$ actually independent of $y_{0}$ and $t_{0}$.

Assuming the validity of Lemmata 1 and 2 we are now going to prove part (b) of Theorem 1. Let $U$ be a compact neighborhood of $A$ in $X$ and $h: U \backslash A \approx$ $\partial U \times[0, \infty[$ a homeomorphism with the properties of Lemma 2, and let $\mu$ be 
a flow on $[0, \infty[$ satisfying the conditions of Lemma 1 . Now we define a flow $X \times \mathbb{R} \rightarrow X$ as follows:

$$
x^{t}:= \begin{cases}x & \text { for } x \in X \backslash U \text { or } x \in A, \\ h^{-1}(y, \mu(s, t)) & \text { for } x \in U \backslash A \text { with }(y, s)=h(x) .\end{cases}
$$

We have to show that our flow is continuous at each point $(x, t) \in X \times \mathbb{R}$. This is clear for $x \in U^{\circ} \backslash A$ or $x \in X \backslash U$. It is continuous on $\partial U$, because in this case we have $s=0$, so that our flow is stationary on $\partial U$. The case $x \in A$ remains. Let $d$ be a metric on $X$. We consider $\varepsilon>0$ and set $\eta:=|t|+1$. Let $\beta>0$ be a number with $|\mu(s, u)-s|<\varepsilon / 2 K$ for $s>\beta$ and $|u|<\eta$; it exists by condition (c) in Lemma 1 . We set $V$ equal to the intersection of the $\varepsilon / 2$-ball around $x$ with the complement of $h^{-1}(\partial U \times[0, \beta]) . \quad V$ is a neighborhood of $x$, and we claim that for each $z \in V$ and $|u|<\eta$ we have $d\left(x, z^{u}\right) \leq \varepsilon$, thus proving continuity of our flow. For $z=h^{-1}(y, s)$ with $y \in \partial U$ and $s>\beta$ we get $d\left(z, z^{u}\right)=d\left(h^{-1}(y, s), h^{-1}(y, \mu(s, u))\right)<\varepsilon / 2$ and hence $d\left(x, z^{u}\right) \leq d(x, z)+d\left(z, z^{u}\right) \leq \varepsilon$.

Finally condition (b) of Lemma 1 implies that $A$ is an attractor of our flow with $U \backslash h^{-1}(\partial U \times[0, \eta])$ as domain of attraction, where $\eta$ is any strictly positive number.

Proof of Lemma 1. We consider the homeomorphism $h:[0, \infty[\approx[0, \infty[$, $h(x)=\ln (1+\ln (1+x))$. It has the property

$$
|h(y)-h(x)| \leq\left|\ln \frac{\ln y}{\ln x}\right| \text { for } x, y \geq e .
$$

Our flow $\mu$ on $\left[0, \infty\left[\right.\right.$ is defined by $\mu(x, t):=h\left(h^{-1}(x) \cdot e^{t}\right)$. Condition (2) translates to:

$$
|\mu(x, t)-x| \leq\left|\ln \left(1+\frac{t}{\ln y}\right)\right|
$$

for $\ln y \geq \max (1,1-t), y=h^{-1}(x) . \mu$ satisfies all requirements.

Proof of Lemma 2. Since $\partial U$ is compact, we can immediately take $V_{0}=\partial U$ and $K\left(y_{0}, t_{0}\right)$ independent of $y_{0}$, and using a suitable partition of unity on $\left[0, \infty\right.$ [ we can furthermore assume that $K\left(t_{0}\right)$ depends continuously on $t_{0}$. Then we define homeomorphisms $\varphi^{-1}:\left[0, \infty\left[\approx\left[0, \infty\left[\right.\right.\right.\right.$ and $g^{-1}: \partial U \times$ $[0, \infty[\approx U \backslash A$ by

$$
\begin{aligned}
\varphi^{-1}(t) & :=\int_{0}^{t} K(s) d s, \\
g^{-1}(y, t) & :=h^{-1}(y, \varphi(t)) .
\end{aligned}
$$

We claim $d\left(g^{-1}(y, t), g^{-1}(y, s)\right) \leq 2(t-s)$ for all $y \in \partial U$ and $s \leq t$. For a proof we take a subdivision $s=t_{0}<t_{1}<\cdots<t_{n}=t$ of $[s, t]$ such that $J_{i}:=\left[\varphi\left(t_{i-1}\right), \varphi\left(t_{i}\right)\right]$ is contained in $W\left(\varphi\left(t_{i}\right)\right)$ and $K(\tau) \geq \frac{1}{2} K\left(\varphi\left(t_{i}\right)\right)$ for $\tau \in J_{i}, i=1, \ldots, n$. We get

$$
t_{i}-t_{i-1}=\int_{\varphi\left(t_{-1}\right)}^{\varphi\left(t_{i}\right)} K(\tau) d \tau \geq \frac{1}{2} \cdot K\left(\varphi\left(t_{i}\right)\right) \cdot\left(\varphi\left(t_{i}\right)-\varphi\left(t_{i-1}\right)\right)
$$


and hence

(7) $d\left(g^{-1}\left(y, t_{i-1}\right), g^{-1}\left(y, t_{i}\right)\right) \leq K\left(\varphi\left(t_{i}\right)\right) \cdot\left(\varphi\left(t_{i}\right)-\varphi\left(t_{i-1}\right)\right) \leq 2\left(t_{i}-t_{i-1}\right)$.

We conclude: $d\left(g^{-1}(y, s), g^{-1}(y, t)\right) \leq \sum_{i=1}^{n} d\left(g^{-1}\left(y, t_{i-1}\right), g^{-1}\left(y, t_{i}\right)\right) \leq$ $2(t-s)$.

Now we will use shape theory to draw conclusions from Theorem 1. A continuous map $f: X \rightarrow Y$ is a shape equivalence, if for each map $\varphi: X \rightarrow P$ into an ANR-space there is $\psi: Y \rightarrow P$ with $\psi f \simeq \varphi$, and if any two such maps $\psi$ are homotopic. Here an ANR-space is a metrizable space $P$, such that any map $f: A \rightarrow P$ defined on a closed subset $A$ of a metrizable space $X$ has an extension over a neighborhood of $A$ in $X$. One particularly interesting property of shape equivalences $f: X \rightarrow Y$ is that the induced homomorphism of Čech cohomology groups $\check{H}^{*}(f): \check{H}^{*}(Y) \rightarrow \check{H}^{*}(X)$ is always an isomorphism. A space $X$ has the shape of a polyhedron $P$ if there exists a shape equivalence $f: X \rightarrow P$. We refer to [8] as a general reference for shape theory.

By a manifold we mean a topological manifold in the sense of [13, Chapter $6, \S 2]$. Every manifold is a separable metrizable space, and by [4, Chapter IV, Proposition 8.12] it is an ANR.

Corollary 1. Every attractor $A$ of a flow on a manifold $X$ has the shape of a finite polyhedron. $A$ has only finitely many components. If in addition $X$ is connected, then $X \backslash A$ has only finitely many components.

Proof. Let $U$ be a neighorhood of $A$ with the properties from Theorem 1(a) and set $V:=U \backslash h^{-1}(\partial U \times[0,1[)$. Clearly $V$ is a compact neighborhood of $A$ in $X$ and $V$ is a retract of the interior of $U$; hence, since manifolds are ANR-spaces, $V$ is a compact ANR. By a conjecture of Borsuk, proved by West [14] $V$ has the homotopy type of a finite polyhedron $P$. (See [8, Appendix 1, $\S 2.2$, Theorem 7] for a discussion of the conjecture and further references.)

We want to show that the inclusion map $A \hookrightarrow V$ is a shape equivalence. ${ }^{1}$ The sets $V_{n}:=U \backslash h^{-1}(\partial U \times[0, n[)$ form a fundamental system of neighborhoods of $A$ in $X$, and each $V_{n}$ is a strong deformation retract of $V$. A retraction $\varrho_{n}: V \rightarrow V_{n}$ can be defined by $\varrho_{n} h^{-1}(x, t):=h^{-1}(x, n)$ for $t \leq n$ and $\varrho_{n}=$ id on $V_{n}$, and a homotopy $D_{n}:$ id $\simeq \varrho_{n}$ relative $V_{n}$ by $D_{n}\left(h^{-1}(x, t), s\right):=$ $h^{-1}(x, s n+(1-s) t)$ for $t \leq n$ and $D_{n}(x, s)=x$ on $V_{n}$. Now we suppose a map $\varphi: A \rightarrow P$ into an ANR-space is given. For some $n$ there is an extension $\varphi^{\prime}: V_{n} \rightarrow P$ of $\varphi$ over $V_{n}$, and $\psi:=\varphi^{\prime} \varrho_{n}: V \rightarrow P$ is an extension of $\varphi$ over the entire set $V$. If $t w o$ maps $\psi, \psi^{\prime}: V \rightarrow P$ into an ANR-space and a homotopy $\Phi: \psi_{\mid A} \simeq \psi_{\mid A}^{\prime}$ are given, then this leads to a map $A \times I \cup V \times \dot{I} \rightarrow P$ equal to $\Phi$ on $A \times I$ and equal to $\psi, \psi^{\prime}$ respectively on $V \times \dot{I}$. This map being defined on a closed subset of the metrizable space $V \times I$ can be extended over a neighborhood and thus provides us with a homotopy $\Phi^{\prime}: \psi_{\mid V_{n}} \simeq \psi_{\mid V_{n}}^{\prime}$ on a suitable $V_{n}$. Then

$$
\Psi(x, s):= \begin{cases}\psi D_{n}(x, 3 s) & \text { for } s \leq \frac{1}{3} \\ \Phi^{\prime}\left(\varrho_{n}(x), 3 s-1\right) & \text { for } \frac{1}{3} \leq s \leq \frac{2}{3} \\ \psi^{\prime} D_{n}(x, 3-3 s) & \text { for } s \geq \frac{2}{3}\end{cases}
$$

\footnotetext{
${ }^{1}$ In fact it is a cylinder base embedding in the sense of Mrozik [9, p. 167].
} 
is a homotopy connecting $\psi$ and $\psi^{\prime}$ on $V$, and $A \hookrightarrow V$ is shown to be a shape equivalence.

Since the number of components is shape invariant (they are counted by 0-dimensional Čech cohomology), $A$ and $P$ have the same number of components. If $X$ is a connected manifold of dimension $n$, then by the Alexander duality theorem [13, Chapter $6, \S 2$, Theorem 17] the singular homology group $H_{1}\left(X, X \backslash A ; \mathbb{Z}_{2}\right)$ with coefficients in $\mathbb{Z}_{2}$ is isomorphic to the Cech cohomology group $\check{H}^{n-1}\left(A ; \mathbb{Z}_{2}\right)$, and since $A$ has the shape of a finite polyhedron it is finitely generated. From the exact homology sequence of the pair $(X, X \backslash A)$ we see that $H_{0}\left(X \backslash A ; \mathbb{Z}_{2}\right)$ is an extension of a quotient of $H_{1}\left(X, X \backslash A ; \mathbb{Z}_{2}\right)$ by a subgroup of $H_{0}\left(X ; \mathbb{Z}_{2}\right) \approx \mathbb{Z}_{2}$ and hence it is finitely generated too, i.e., $X \backslash A$ has finitely many components.

Corollary 1 shows that although attractors may be complicated spaces they cannot be too irregular. Let us take a look at some

Examples. 1. The Warsaw circle (i.e., the graph of the function $x \mapsto \sin 1 / x$ for $0<x \leq 1 / \pi$ including the limit segment on the ordinate axis and an arc joining the endpoint of this segment to $(1 / \pi, 0))$ is an attractor of a flow on $\mathbb{R}^{2}$, as mentioned by Hastings [6, Example 3.3]. Despite its bad local properties the Warsaw circle has the shape of an ordinary circle.

2. The Hawaiian earring (i.e., the union of a sequence of circles $A_{n}=$ $\left\{(x, y) \in \mathbb{R}^{2} \mid x^{2}+(y-1 / n)^{2}=1 / n^{2}\right\}$ of decreasing diameter converging to a common point on their boundary) cannot be an attractor of a flow on a manifold, because its first Čech cohomology group is not finitely generated and hence it does not have the shape of a finite polyhedron.

3. The solenoid (i.e., the inverse limit of a sequence of circles and bonding maps of degree two) cannot be an attractor of a flow on a manifold for the same reason as in Example 2. But it is well known that it is an attractor of a cascade on a 3-dimensional manifold: In $[12, \S 4]$ there is described an embedding $h: D^{2} \times S^{1} \longmapsto D^{2} \times S^{1}$ of the 3 -dimensional solid torus into itself such that the inverse sequence consisting of solid tori as terms and $h$ as bonding maps has the solenoid as the inverse limit. We observe that $\operatorname{im} h$ is contained in the interior of the torus. Hence, if $X$ is the limit of the direct sequence with solid tori as terms and $h$ as bonding maps, then $X$ is covered by open tori and consequently it is a 3-dimensional manifold. $h$ induces an embedding $g: X \mapsto X$ into itself, which is surjective and hence a homeomorphism. The generated cascade on $X$ has the solenoid as an attractor.

For any self-map $f: X \rightarrow X$ of a space $X$ we denote by $T(f)$ its mapping torus, i.e., the space obtained from $X \times I$ by identifying the points $(x, 0)$ and $(f(x), 1)$.

Corollary 2. We suppose that $A$ is an attractor of a cascade $\mu: X \times \mathbb{Z} \rightarrow X$ on a manifold $X$ and denote by $f: X \approx X$ the generating homeomorphism $f(x):=\mu(x, 1)$. Then $T\left(f_{\mid A}\right)$ has the shape of a finite polyhedron. ${ }^{2}$

We observe that Corollary 2 puts restrictions on the homeomorphism generating a cascade with prescribed attractor $A$ : It can be stationary on $A$ only if

\footnotetext{
${ }^{2}$ This interesting observation was contributed by the referee.
} 
$A \times S^{1}$ has the shape of a finite polyhedron. There exist compacta $A$ such that $A \times S^{1}$, but not $A$ itself has the shape of a finite polyhedron [8, Chapter II, $\S 9.5$, Example 2 and Remark 6].

Proof. We consider the manifold $Y:=T(f)$ and the flow on $Y$ defined by $[x, s]^{t}:=\left[f^{n}(x), s-t+n\right]$ for $n-1 \leq t-s \leq n . T\left(f_{\mid A}\right)$ is an attractor of this flow and hence it must have the shape of a finite polyhedron.

In the case of Example $3 f_{\mid A}$ is the shift map [12, Lemma 29] of the solenoid $A$. Let $\varphi: S^{1} \rightarrow S^{1}$ be a map of degree 2 . Then $T\left(f_{\mid A}\right)$ has the shape of $T(\varphi)$. The latter is a CW-complex consisting of a wedge of two circles $a$ and $b$ and a 2-cell attached by the loop $a b a^{-1} b^{-2}$.

Corollary 3. A compact subset of the plane is an attractor of a suitable flow on the plane if and only if it has the shape of a finite union of finite wedges of circles or, equivalently, if and only if its Čech cohomology groups are finitely generated.

For the proof we need the following lemma:

Lemma 3. An open, connected subset $U$ of the plane with trivial first homology group is diffeomorphic to a disc.

Proof. It suffices to show that $U$ is simply connected; then the Riemann mapping theorem applies. Let $\omega: S^{1} \rightarrow U$ be a loop in $U$. We may suppose that $\omega$ is piecewise linear and then we can decompose it in finitely many simple parts, so that $\omega$ itself may be assumed to be simple. If $V$ is the bounded component of $\mathbb{R}^{2} \backslash \omega\left(S^{1}\right)$, then the Jordan-Schoenflies theorem [15, p. 94, Theorem 5.9] ensures the existence of a homeomorphism $V \cup \omega\left(S^{1}\right) \approx D^{2}$ onto the closed unit disc $D^{2}$ mapping $V$ onto its interior. Since $\omega$ is null homologous in $U$, the set $V \cup \omega\left(S^{1}\right)$ must be entirely in $U$; but then $\omega$ is evidently null homotopic.

Proof of Corollary 3. If $A$ is an attractor of a flow on the plane, then by Corollary 1 it has the shape of a finite polyhedron and by [8, p. 201, Theorem 5 and p. 203, Remark 2] each of its finitely many components has the shape of a finite wedge of circles.

If $A$ is of such shape, then its Čech cohomology groups are finitely generated.

Now let us assume that the Čech cohomology groups of $A$ are finitely generated; we want to construct a neighborhood $U$ satisfying the assumptions of Theorem 1(b). The cohomology group in dimension 0 tells us particularly that $A$ has only finitely many components, so that we may assume $A$ to be connected. We add an infinite point to the plane turning it into $S^{2}$. As in the proof of Corollary 1 , observing that $\breve{H}^{1}(A ; \mathbb{Z})$ is finitely generated we see that $S^{2} \backslash A$ has only finitely many components. The Alexander Duality Theorem [13, Chapter 6, $\S 2$, Theorem 17] implies that the homomorphism $\mathrm{H}_{2}\left(S^{2} ; \mathbb{Z}\right) \rightarrow \mathrm{H}_{2}\left(S^{2}, S^{2} \backslash A ; \mathbb{Z}\right)$ induced by the inclusion map is isomorphic to $\check{H}^{0}\left(S^{2} ; \mathbb{Z}\right) \rightarrow \check{H}^{0}(A ; \mathbb{Z})$ and hence to the identity map of $\mathbb{Z}$. From the exact homology sequence

$$
H_{2}\left(S^{2}\right) \approx H_{2}\left(S^{2}, S^{2} \backslash A\right) \rightarrow H_{1}\left(S^{2} \backslash A\right) \rightarrow H_{1}\left(S^{2}\right)=0
$$


we conclude $H_{1}\left(S^{2} \backslash A\right)=0$. Of course every component of $S^{2} \backslash A$ must have trivial first homology too, and now Lemma 3 tells us that each such component is diffeomorphic to a disc. Taking a smaller open disc out of it we arrive at a neighborhood $U$ of $A$ of the required form. The local Lipschitz condition (L) is a consequence of continuous differentiability.

In higher dimensions the situation is much more complicated. We do not know if the homeomorphism type of a compactum in $\mathbb{R}^{n}, n>2$, is sufficient to give a characterization similar to Corollary 3 ; we have to add embedding conditions. The key to the problem is Chapman's finite-dimensional complement theorem [3]:

Theorem (Chapman). We consider two compact subsets $X, Y \subset \mathbb{R}^{n}$ in standard position with $n \geq 2+2 \max (\operatorname{dim} X, \operatorname{dim} Y)$. If $X$ and $Y$ are shape equivalent, then there exists a PL-isomorphism $\mathbb{R}^{n} \backslash X \approx \mathbb{R}^{n} \backslash Y$ that can be extended to a homeomorphism $\mathbb{R}^{n} / X \approx \mathbb{R}^{n} / Y$.

Chapman's paper does not contain the theorem in this formulation, but it comes out of his proof. The homeomorphism of $\mathbb{R}^{n} / X$ and $\mathbb{R}^{n} / Y$ was first explicitly observed by Geoghegan and Summerhill [5]. Standard position is defined in [3, Definition 3.1]. We observe that any subpolyhedron of $\mathbb{R}^{n}$ is in standard position.

Theorem 2. Any finite-dimensional compactum $X$ of finite polyhedral shape can be embedded in $\mathbb{R}^{n}$ for suitable $n$ in such a way that there is a flow on $\mathbb{R}^{n}$ having $X$ as an attractor.

Corollary 4. A finite-dimensional compactum can be an attractor of a continuous flow on a manifold if and only if it has the shape of a finite polyhedron.

Proof. To start let us assume that $Y$ is a finite subpolyhedron of some $\mathbb{R}^{n}$, and let us consider a triangulation of $\mathbb{R}^{n}$ such that $Y$ actually corresponds to a full subcomplex. By $\psi_{p}: \mathbb{R}^{n} \rightarrow I$ we denote the barycentric coordinate function with respect to a vertex $p$, and by $K$ we denote the set of vertices of $Y$. We set

$$
\varphi:=\sum_{p \notin K} \psi_{p}: \mathbb{R}^{n} \rightarrow I
$$

Then $Y=\varphi^{-1}(0)$, hence $V:=\varphi^{-1}\left[0, \frac{1}{2}\right]$ is a closed neighborhood of $Y$ with $\partial V=\varphi^{-1}\left(\frac{1}{2}\right) . \quad V$ is contained in the star of $Y$ with respect to our triangulation, which is necessarily locally finite; therefore, $V$ is compact. We define a homeomorphism $\vartheta=\left(\vartheta_{1}, \vartheta_{2}\right): V \backslash Y \approx \partial V \times[0, \infty[$ by

$$
\begin{gathered}
\vartheta_{1}:=\frac{1}{2} \sum_{p \notin K} \frac{\psi_{p}}{\varphi} p+\frac{1}{2} \sum_{p \in K} \frac{\psi_{p}}{1-\varphi} p, \\
\vartheta_{2}:=\frac{1}{\varphi}-2 .
\end{gathered}
$$

Its inverse is given by

$$
\vartheta^{-1}(y, t):=\sum_{p \notin K} \frac{2 \psi_{p}(y)}{t+2} p+\sum_{p \in K} 2 \frac{t+1}{t+2} \psi_{p}(y) p,
$$


and since this is continuously differentiable with respect to $t$, it satisfies the Lipschitz condition (L).

Now we suppose that $X$ is a finite-dimensional compactum, which is shape equivalent to $Y$. By [3, Proposition 3.4] $X$ can be embedded in some $\mathbb{R}^{n}$, such that it has standard position, and we may furthermore assume $n \geq 2+$ $2 \max (\operatorname{dim} X, \operatorname{dim} Y)$. By Chapman's theorem there is a PL-homeomorphism $g: \mathbb{R}^{n} \backslash X \approx \mathbb{R}^{n} \backslash Y$ extending to a homeomorphism $\mathbb{R}^{n} / X \approx \mathbb{R}^{n} / Y$. Then $U:=\mathbb{R}^{n} \backslash g^{-1}\left(\mathbb{R}^{n} \backslash V\right)$ is a compact neighborhood of $X$, because $U / X \approx V / Y$ is a compact neighborhood of the basepoint in $\mathbb{R}^{n} / X . g$ maps $\partial U$ onto $\partial V$. Since $g$ is piecewise linear, it is Lipschitz continuous; therefore, the composed homeomorphism $h:=\left(g^{-1} \times \mathrm{id}\right) \vartheta g: U \backslash X \approx \partial U \times[0, \infty$ [ satisfies condition (L). Now Theorem 1(b) provides us with a flow on $\mathbb{R}^{n}$ having $X$ as an attractor.

Corollary 4 follows from Theorem 2 and Corollary 1 .

Remark. The flow constructed in the above proof is always stationary on the attractor. This it not surprising: An arbitrary flow can be altered to achieve this form by slowing down the motion on the complement of the attractor. The reader should observe:

(1) There exist compacta $A$ of trivial shape, such that every path component of $A$ consists of a single point, for instance, the pseudoarc [2]. Such a compactum can be an attractor, but every flow on $A$ is necessarily stationary.

(2) The situation is different for cascades: If a cascade $\mu: X \times \mathbb{Z} \rightarrow X$ on a manifold $X$ has a solenoid $A$ as attractor, then it follows form Corollary 2 that $\mu$ cannot be stationary on $A$.

Problems. (1) Is it possible to charactarize those compacta $A$ that can be attractors of flows on manifolds such that $A$ contains a dense orbit?

(2) Is it possible to characterize attractors of cascades?

\section{ADDED IN PROOF}

After the authors finished their work on this paper they learned of the existence of the article On the structure of attracting compacta by S. A. Bogatyj and V. I. Gutsu in Differentsial'nye Uravneniya 25 (1989), 907-909 (Russian), which contains part of the results presented here.

\section{REFERENCES}

1. D. V. Anosov and V. I. Arnold, Dynamical systems. I, Encyclopedia Math. Sci., vol. 1, Springer, New York, 1988.

2. R. H. Bing, Concerning hereditarily indecomposable continua, Pacific J. Math. 1 (1951), 43-51.

3. T. A. Chapman, Shapes of finite-dimensional compacta, Fund. Math. 76 (1972), 261-276.

4. A. Dold, Lectures on algebraic topology, Grundlehren Math. Wiss., vol. 200, Springer. New York and Berlin, 1980.

5. R. Geoghegan and R. R. Summerhill, Concerning the shapes of finite-dimensional compacta. Trans. Amer. Math. Soc. 179 (1973), 281-292.

6. H. M. Hastings, A higher dimensional Poincaré-Bendixson theorem, Glas. Mat. 14 (1979). 263-268.

7. M. W. Hirsch and S. Smale, Dijferential equations, dynamical systems, and linear algehra, Academic Press, New York, 1974. 
8. S. Mardešić and J. Segal, Shape theory, Mathematical Library, vol. 26, North-Holland, Amsterdam, 1982.

9. P. Mrozik, Mapping cylinders of approaching maps and strong shape, J. London Math. Soc. (2) 41 (1990), 159-174.

10. J. W. Robbin and D. Salamon, Dynamical systems, shape theory and the Conley index, Ergodic Theory Dynamical Systems 8* (1988), 375-393.

11. D. Salamon, Connected simple systems and the Conley index of isolated invariant sets, Trans. Amer. Math. Soc. 291 (1985), 1-41.

12. R. M. Schori, Chaos: An introduction to some topological aspects, Continuum Theory and Dynamical Systems (Morton Brown, ed.), Contemp. Math., vol. 117, Amer. Math. Soc., Providence, RI, 1991, pp. 149-161.

13. E. H. Spanier, Algebraic topology, McGraw-Hill, New York, 1966.

14. J. E. West, Mapping Hilbert cube manifolds to ANR's: A solution to a conjecture of Borsuk, Ann. of Math. (2) 106 (1977), 1-18.

15. R. L. Wilder, Topology of manifolds, Colloq. Publ., vol. 32, Amer. Math. Soc., Providence, RI, 1949.

Fachbereich Mathematik, Johann Wolfgang Goethe-Universität, Robert-MayerSTRASSE 6-10, 6000 Frankfurt, GermanY

Current address: Helpertseestraße 21, 6052 Mühlheim, Germany

E-mail address: 100034.3630@CompuServe.COM

Department of Mathematics, University of Washington, Seattle, Washington 98195

E-mail address: segal@math.washington.edu 